\title{
Toughening Hydrogels Through Force-triggered Chemical Reactions that Lengthen Polymer Strands
}

Authors: Zi Wang ${ }^{1,2}$, Xujun Zheng ${ }^{1}$, Tetsu Ouchi ${ }^{1,2}$, Tatiana B. Kouznetsova ${ }^{1,2}$, Haley K. Beech $^{2,5}$, Sarah Av-Ron ${ }^{5}$, Brandon H. Bowser ${ }^{1,2}$, Shu Wang ${ }^{1,2}$, Jeremiah A. Johnson ${ }^{2,3}$, Julia A.

Kalow $^{2,4^{*}}$, Bradley D. Olsen ${ }^{2,5^{*}}$, Jian Ping Gong ${ }^{2,6,7,8^{*}}$, Michael Rubinstein ${ }^{1,2,6,8,9^{*}}$, and Stephen L. Craig $^{1,2,6^{*}}$

\author{
Affiliations: \\ ${ }^{1}$ Department of Chemistry, Duke University, Durham, NC, USA 27708-0346 \\ ${ }^{2}$ NSF Center for the Chemistry of Molecularly Optimized Networks \\ ${ }^{3}$ Department of Chemistry, MIT, Boston, MA, USA \\ ${ }^{4}$ Department of Chemistry, Northwestern University, Evanston, IL, USA \\ ${ }^{5}$ Department of Chemical Engineering, MIT, Boston, MA, USA \\ ${ }^{6}$ Soft Matter GI-CoRE, Hokkaido University, N21W11, Kita-ku, Sapporo 001-0021, Japan \\ ${ }^{7}$ Faculty of Advanced Life Science, Hokkaido University, N21W11, Kita-ku, Sapporo 001-0021, \\ Japan \\ ${ }^{8}$ Institute for Chemical Reaction Design and Discovery, Hokkaido University, N21W11, Kita-ku, \\ Sapporo 001-0021, Japan \\ ${ }^{9}$ Department of Mechanical Engineering and Materials Science, Biomedical Engineering, and \\ Physics, Duke University, Durham, NC, USA \\ *Correspondence to: jkalow@northwestern.edu, jaj2109@mit.edu, bdolsen@mit.edu, \\ gong@sci.hokudai.ac.jp, michael.rubinstein@duke.edu, stephen.craig@duke.edu
}




\begin{abstract}
The utility and lifetime of materials made from polymer networks, including hydrogels, depend on their capacity to stretch and resist tearing. In gels and elastomers, those mechanical properties are often limited by the covalent chemical structure of the polymer strands between cross-links, which is typically fixed during the material synthesis. Here, we report polymer networks in which the constituent strands lengthen through force-coupled reactions that are triggered as the strands reach their nominal breaking point. Reactive strand extensions of up to $40 \%$ lead to hydrogels that stretch $40-50 \%$ further than, and exhibit tear energies twice that of, networks made from analogous control strands. The enhancements are synergistic with those provided by double network architectures, and complement other existing toughening strategies.
\end{abstract}




\section{Main Text:}

Polymeric materials such as elastomers gradually break down under repeated use. This process of physical fatigue is due to a series of microscopic tearing events that occur during periods of mechanical strain $(1,2)$, and it limits the useful lifetime of products ranging from automobile tires to prosthetic implants, and from stretchable displays to soft robots and other devices. Polymer network fatigue therefore contributes to a wide range of undesirable endpoints, including the rate at which commodity rubber products end up in landfills (ca. $2.5 \times 10^{8}$ tires/yr in the U.S. alone) (3), the number of replacement surgeries due to failing implants such as artificial knees $(4,5)$, and the limits to which flexible displays can be stretched before breaking (6). As a result, recent years have seen extensive effort given to strategies that improve the durability of polymer networks $(7,8)$, including the use of multi-network architectures $(7,9-11)$, reversible cross-linking $(10,12)$, noncovalent domain unfolding (13-16), and the addition of fillers (17). All of these approaches have in common that the primary, covalent chemical structure of the network remains fixed up until the point that the polymer strand breaks.

Polymer networks that actively remodel their primary, covalent structure during use therefore have the potential to provide property enhancements that complement existing mechanisms. Recent advances in covalent polymer mechanochemistry now enable the synthesis of polymer strands that extend the covalent bonding along their backbone when stretched (18), as a result of force-coupled chemical reactions that are triggered at or just before the strands reach their nominal breaking point. Because these reactions are typically associated with high forces $(\sim \mathrm{nN})$, the amount of energy absorbed at the single molecular level can be quite large relative to the energy absorbed by a covalent strand in the absence of the reaction-enabled extension (19-21). Existing models of network fracture draw a direct relationship between the energy required to propagate a crack through a rubbery material (the tear energy, $T$ ) and the energy required to stretch an individual polymer strand to the point at which it breaks (the chain fracture energy, $U$ ) $(22,23)$. We therefore hypothesized that by turning a polymer network into a literal network of responsive chemical reactions, reactive strand extension might improve the properties of bulk materials. 
A Toughening DN by reactive strand extension

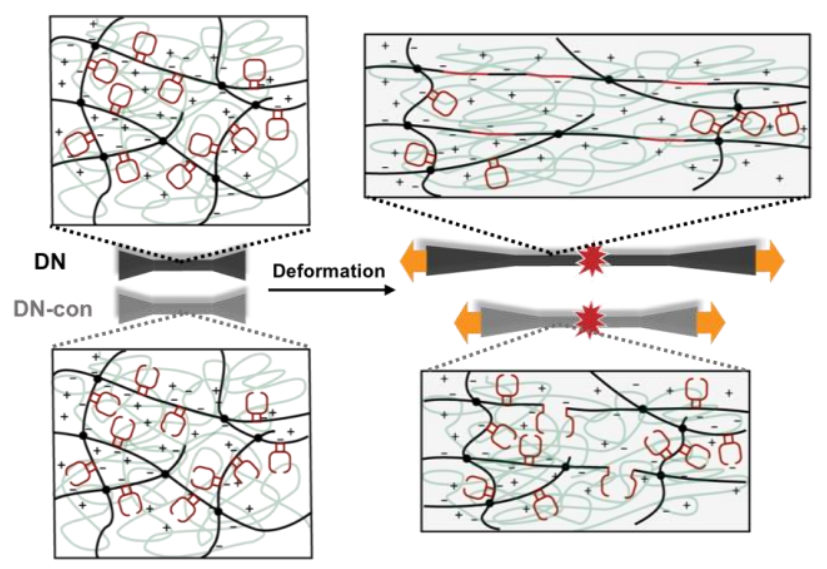

B Chemical designs of polyelectrolytes in first network

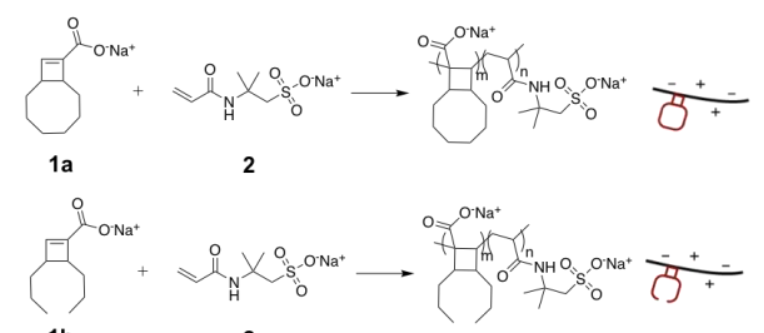

1b

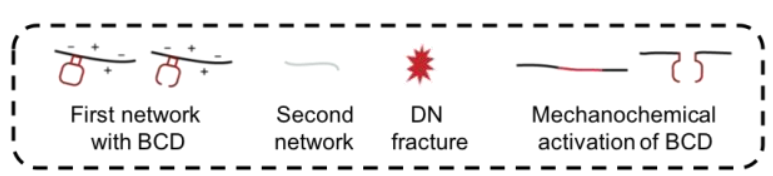

Fig. 1. The reactive strand extension (RSE) concept in double network hydrogels (strand scission events are not shown). (A) Schematic of double networks RSE and an analogous control polymer. (B) Chemical structures of the first network co-polymers employed in this work.

Here we demonstrate that reactive strand extension (RSE) can be used to improve the stretchability and tear resistance of hydrogels. The RSE concept is shown in Figure 1, and the strand extension is provided by the sodium salts of bicyclo[6.2.0]decane (BCD) mechanophores, which react via a force-coupled [2+2] cycloreversion to release stored length (24). BCD copolymers are formed via the radical addition co-polymerization of the corresponding bicyclic cyclobutene carboxylate monomer 1a. The mechanochemical activity of the sodium salts of BCD was quantified using single-molecule force spectroscopy (SMFS) implemented through an atomic force microscope (AFM). Stretching of the single molecules to the forces required for BCD cycloreversion requires strong attachments of the polymer to the AFM tip, and we found that an acetoacetate group could be introduced to promote rapid and strong bonding to aminefunctionalized AFM tips $(25,26)$. Co-polymerization of 1a with acrylamido-2-methylpropane sulfonate (NaAMPS) $\mathbf{2}$ and acetoacetate methacrylate $\mathbf{3}$ provided linear co-polymers P1 with 1a:2:3 $=0.22: 0.62: 0.16$. At forces of up to $\sim 1500 \mathrm{pN}$, the SMFS curves are typical of a flexible polymer strand, as extension initially requires only low forces (order of $1-10 \mathrm{pN}$ ) to overcome the conformational entropy associated with bond torsions along the polymer main chain. As the extension increases, the available conformational degrees of freedom are exhausted and the trapped polymer strand reaches end-to-end distances that approach its fully extended contour length (order of $10^{2} \mathrm{pN}$ ). Subsequent stretching is dominated by the deformation of bond angles 
and bond lengths, and only small fractional extensions are achieved with increasing force, as the polymer nears its physical stretching limits before breaking (order of $10^{3} \mathrm{pN}$ ). With the BCD mechanophore, however, the force-separation curve shows a reproducible transition across 1500$2000 \mathrm{pN}$, in which the release of stored length of up to $40 \%$ of the initial polymer contour length is observed. This stored length is consistent with that expected from the BCD cycloreversion, and the transition force is similar to that reported in other cyclobutane mechanophores $(20,27,28)$. The onset of extension also provides a measure of the scission point of control strands made from $\mathbf{1 b}$ (no fused ring), and CoGEF calculations (see Fig. S2) confirm that the scission behave of the fused and unfused cyclobutanes are effectively indistinguishable (29). Thus, BCD incorporation provides the desired molecular behavior: as the polymer strands reach their nominal stretching limit, the presence of selective mechanophores converts strand scission events into force-coupled covalent rearrangements that allow the strands to survive forces and extensions that would otherwise be catastrophic.

A Mechanochemical reactions

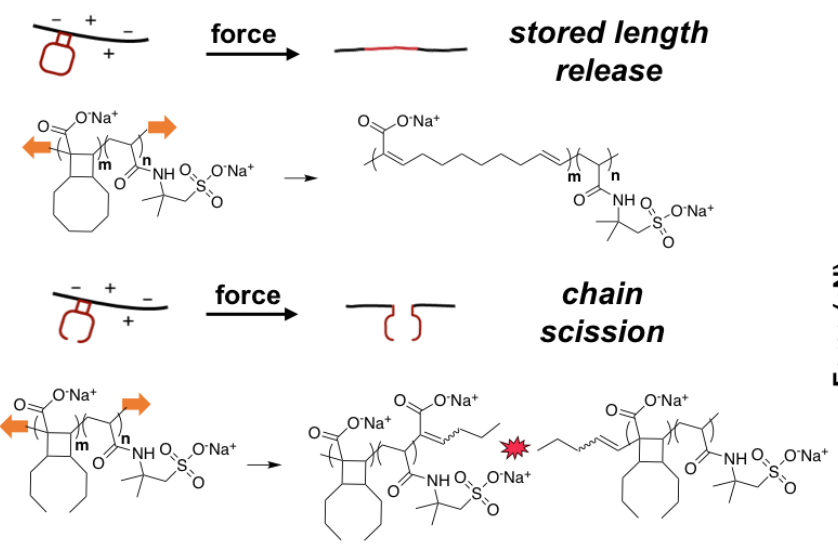

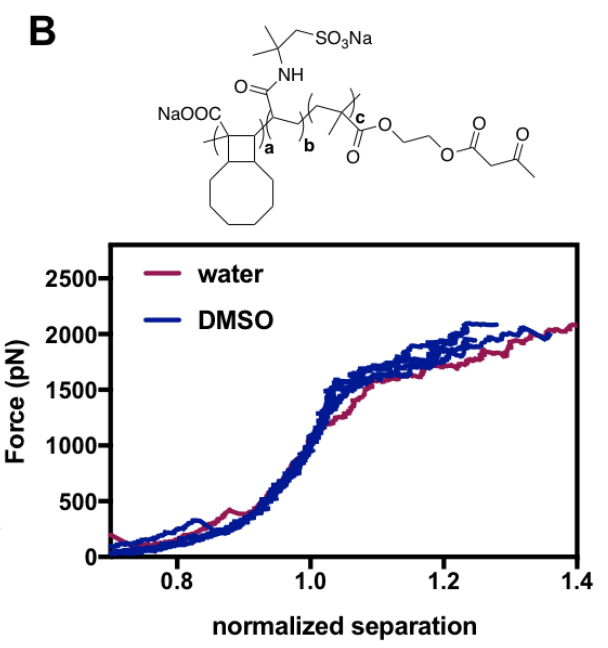

Fig. 2. Mechanochemical reactions driving RSE. (A) Mechanically coupled [2+2] cycloreversion leads to chain extension in [6.2.0]bicyclodecane mechanophores and to chain scission in analogous acyclic cyclobutane mechanophores. (B) Representative single molecule forceextension curves of linear RSE co-polymers in water and DMSO.

To see whether this single molecule behavior has an impact on bulk material properties, the mechanochemically active co-polymers were incorporated into double-network (DN) hydrogels, whose robust mechanical properties facilitate the characterization of mechanical response. A 
DN hydrogel consists of two interpenetrating networks with contrasting structure: the first network comprises strands in a highly stretched state and is rigid and brittle. The second network comprises strands in a coiled state, and as a result it is soft and stretchable $(30,31)$. This doublenetwork structure allows multiple strands within the first brittle network to break independently without causing catastrophic breakdown of the entire brittle network, as the local strain amplification created by the breaking of the brittle strand is suppressed by transferring the load to the soft network strands nearby rather than propagating through the first network (31-34). Hydrophilic polymer networks were made via the radical addition co-polymerization of the sodium salts of BCD 1a, NaAMPS 2 (aqueous feed concentrations of $0.3 \mathrm{M}$ and $0.7 \mathrm{M}$ for $1 \mathrm{a}$ and 2, respectively) and $N, N^{\prime}$-methylenebisacrylamide (MBAA) cross-linker (8 mol\% or $4 \mathrm{~mol} \%$ of total monomer feed ratio). In the DNs, this BCD-AMPS co-polymer was used as the first network, and sparsely cross-linked polyacrylamide PAAm (AAm, 4 M; MBAA, 0.01 mol\%) was used as the soft and stretchable second network. The DN structure is formed by swelling the formed first network in a precursor monomer solution of the second network, followed by polymerization of the second network (see Fig. S1). Two first networks, N1 and N2, of the same BCD-AMPS content $(\mathrm{BCD}: \mathrm{AMPS}=3: 17 \mathrm{~mol} / \mathrm{mol} ; \sim 40 \%$ contour length increase expected from CoGEF calculations, see Fig. S2) but different cross-linking density (8 mol\% and $4 \mathrm{~mol} \%$ of MBAA for N1 and N2, respectively), were prepared (see Table S1 and Fig. S1).

Control networks N1-con and N2-con were synthesized using acyclic cyclobutene carboxylate $\mathbf{1 b}$. The structural similarity of $\mathbf{1 a}$ and $\mathbf{1 b}$ was expected to lead to similar reactivity in the polymerization and, thus, similar network structure in N1 and N1-con, or in N2 and N2con. This expectation was verified in multiple ways. First, the reactivity ratios of $\mathbf{1 a}$ and $\mathbf{1 b}$ with NaAMPS were measured and found to be $r_{1 a}=0.19 \pm 0.01 ; r_{N a A M P S}=1.85 \pm 0.09$ and $r_{1 b}=0.16$ $\pm 0.01 ; r_{\text {NaAMPS }}=1.87 \pm 0.09$, respectively (see Fig. S3). Second, the equilibrium swelling ratios $\left(\mathrm{V} / \mathrm{V}_{0}\right)$ of $\mathbf{N 1}(12.4 \pm 0.1)$ and $\mathbf{N 2}(32.7 \pm 0.7)$ in water are indistinguishable from those of N1con (12.1 \pm 0.9) and N2-con (32.7 \pm 1.8$)$, respectively. Third, N1, N1-con, N2, and N2-con were disassembled through the hydrolysis of the bis-acrylamide cross-linker $\mathbf{4}$, and the gel permeation chromatography (GPC) elugrams of the fragments were found to be very similar (see Fig. S4). Finally, these single networks were too brittle for reliable mechanical characterization, but forming DNs by cross-linking PAAm inside the swollen first networks resulted in moduli of DN1 $(288 \pm 4 \mathrm{kPa})$ and DN1-con $(290 \pm 8 \mathrm{kPa})$ that fall within experimental uncertainty. The 
Table 1. Young's modulus $(E)$, nominal strain, nominal stress, and input energy density at break ( $\varepsilon_{b}, \sigma_{b}$, and $W_{\mathrm{b}}$, respectively) of unnotched films in unconstrained uniaxial tension, and crack propagation strain $\left(\boldsymbol{\varepsilon}_{\boldsymbol{p}}\right)$ and tearing energy $(T)$ of films in a single-edge notched geometry; tearing energy of PAAm gel is listed as previously reported (44).

\begin{tabular}{ccccccc} 
& $\boldsymbol{E}(\mathbf{k P a})$ & $\boldsymbol{\varepsilon}_{\boldsymbol{b}}$ & $\boldsymbol{\sigma}_{\boldsymbol{b}}(\mathbf{M P a})$ & $\boldsymbol{W}_{\mathbf{b}}\left(\mathbf{M J} \mathbf{~ m}^{-\mathbf{3}}\right)$ & $\boldsymbol{\varepsilon}_{\boldsymbol{p}}$ & $\boldsymbol{T}\left(\mathbf{J ~ m}^{-\mathbf{2}}\right)$ \\
\hline $\mathbf{D N 1}$ & $288 \pm 4$ & 10.0 & 0.92 & 6.4 & 2.3 & $2840 \pm 103$ \\
$\mathbf{D N 1 - c o n}$ & $290 \pm 8$ & 4.9 & 0.61 & 2.3 & 1.7 & $1419 \pm 52$ \\
$\mathbf{D N 2}$ & $243 \pm 5$ & 7.0 & 0.64 & 2.6 & 1.3 & $554 \pm 40$ \\
DN2-con & $249 \pm 13$ & 4.7 & 0.54 & 1.6 & 0.8 & $276 \pm 10$ \\
PAAm & $57 \pm 6$ & 5.0 & 0.18 & 0.4 & $/$ & $\sim 10$
\end{tabular}

moduli of DN2 $(243 \pm 5 \mathrm{kPa})$ and DN2-con $(249 \pm 13 \mathrm{kPa})$ are similarly matched. Thus, any differences in ultimate stretch and tear resistance between the active and control networks are attributed to the conversion of scission to molecular extension that is allowed in the BCD copolymers by virtue of the designed release of length stored in the bicyclic ring structure.

The incorporation of the BCD mechanophore leads to substantial changes in the mechanical limits of its double networks. The resistance to tearing was evaluated by introducing a notch into DN films and measuring the strain necessary for the notch to propagate under pure shear loading. Figure 3A, B shows the stress-strain behavior of notched DN1 and DN1-con under uniaxial extension. The critical stretch for crack propagation in DN1 is $234 \pm 4 \%$, which is $40 \%$ greater than the $167 \pm 7 \%$ observed in DN1-con. The gain in stretchability is found as well in DN2, for which the critical stretch for crack propagation is $128 \pm 8 \%$, vs. $83 \pm 2 \%$ in DN2-con (53\% greater strain). The resistance to tearing can be further quantified in terms of the tear energy $T$, or the energy required per area of crack growth, which was determined via the method of Rivlin and Thomas (35). Figure 3D shows that both DN1 and DN2 have roughly double the $T$ of their respective controls: $2,840 \pm 103 \mathrm{~J} / \mathrm{m}^{2}$ vs. $1,419 \pm 52 \mathrm{~J} / \mathrm{m}^{2}$ for DN1 vs. DN1-con, and $554 \pm 40$ $\mathrm{J} / \mathrm{m}^{2}$ vs. $276 \pm 10 \mathrm{~J} / \mathrm{m}^{2}$ for DN2 vs. DN2-con. 
A

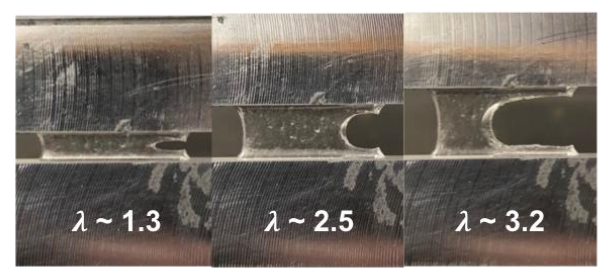

C

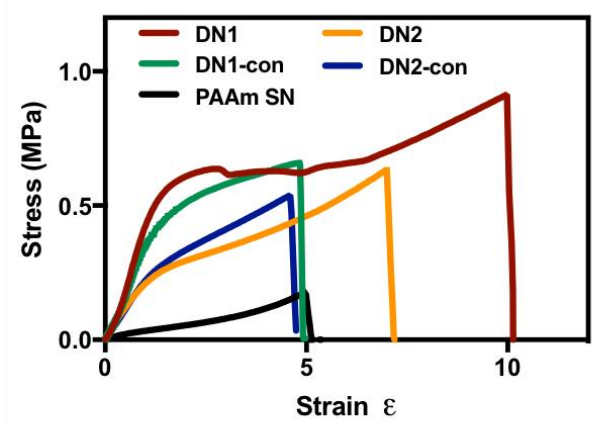

D

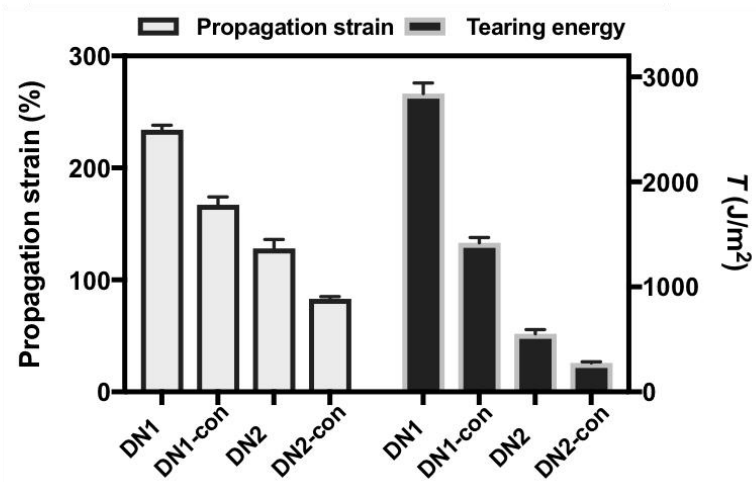

B

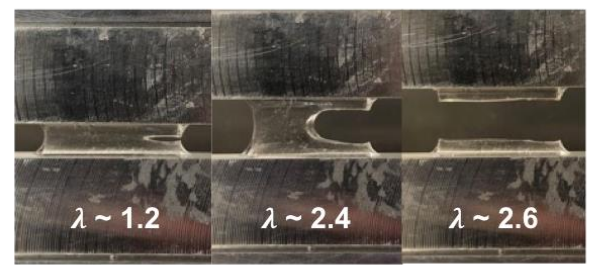

E
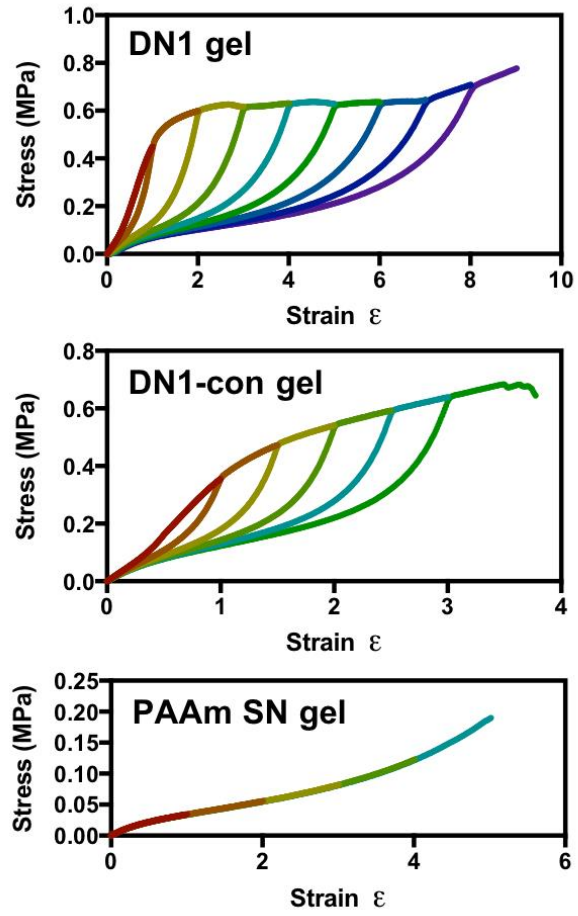

Fig. 3. Response of DNs to uniaxial tension. (A) Single-edge notched DN1 at stretch ratios $\lambda(\mathrm{mm} / \mathrm{mm})$ of $1.3,2.5$, and 3.2. (B) Single-edge notched DN1-con at stretch ratios $(\mathrm{mm} / \mathrm{mm})$ of 1.2, 2.4, and 2.6. (C) Representative stress-strain curves (pure shear, $2 \mathrm{~mm}$ thick films) of unnotched DNs and a single PAAm network prepared identically to the second network of the DNs. (D) Propagation strain and tear energy of DNs. (E) Hysteresis in cyclic loading/unloading of DN1, DN1-con, and a single PAAm network prepared identically to the second network of the DNs.

The improved mechanical properties due to reactive strand extension are also observed in unnotched DNs (see Fig. S5). Figure 3C shows the stress-strain behavior of a pristine DN1 film 
under uniaxial extension. The film survives to an ultimate stretch of $890 \pm 120 \%$ before breaking, whereas the acyclic control DN1-con fails at a lower stretch of $479 \pm 99 \%$. The high strains obtained in the unnotched films reveal that RSE changes the internal fracture patterns of the DN. Most notably, DN1 shows a clear yielding, suggesting that the first network ruptures into a global discontinuous phase that enables the load to be transferred to the second network in the yielded region. On the other hand, DN1-con shows a gradual increase in stress without a clear plateau stress region, suggesting that the internal rupture of the first network is relatively random and does not achieve a global discontinuous structure up to sample failure. We attribute this to the fact that fracture of N1-con within DN1-con begins at lower strain than N1. When a small defect forms, that stress largely remains in N1-con, because the second network is still too soft for stress transfer (the local strain at the defect is not great enough). Thus, the fracture propagates further across N1-con within the DN and only begins to transfer to the second network after the defect becomes larger. In DN1, fracture of N1 initiates at higher strain, at which point a greater fraction of the stress is transferred to the second network, and so the fracture event is blunted and the next fracture occurs elsewhere, leading to the desired fractal/domain patterns for greater toughness (31).

The presence of RSE should alter the energy dissipation behavior from that observed in conventional DN hydrogels, because it can occur through covalent rearrangement without chain scission. Here, we examined the change in modulus and energy dissipated in sequential loading/unloading cycles response under uniaxial tension, where the maximum strain increases from one loading cycle to the next. When a DN gel is stretched to a given ratio for the first time and then unloaded, there is significant hysteresis (33); this typical DN behavior is observed as well in our systems (Figure 3E; low strain behavior expanded in Figure 4A,B). Typically, hysteresis in DN gels is attributed almost entirely to chain scission in the first network (31), and it is therefore correlated with a decrease in modulus due to the loss of stress-bearing network 
A

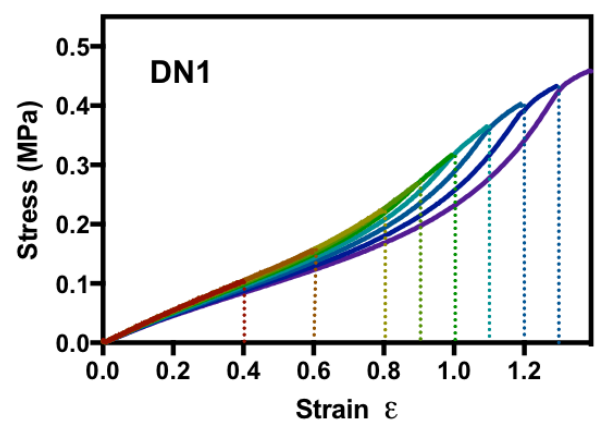

C

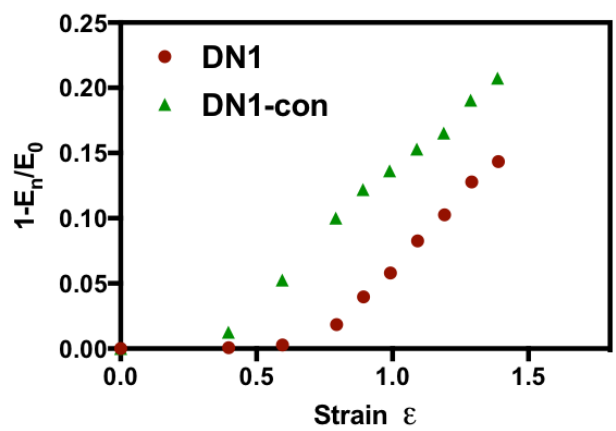

B

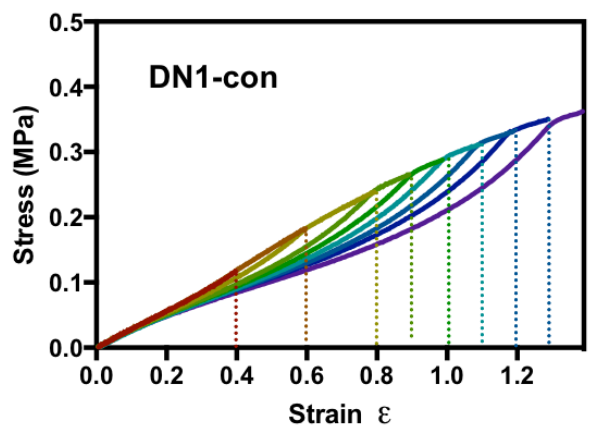

D

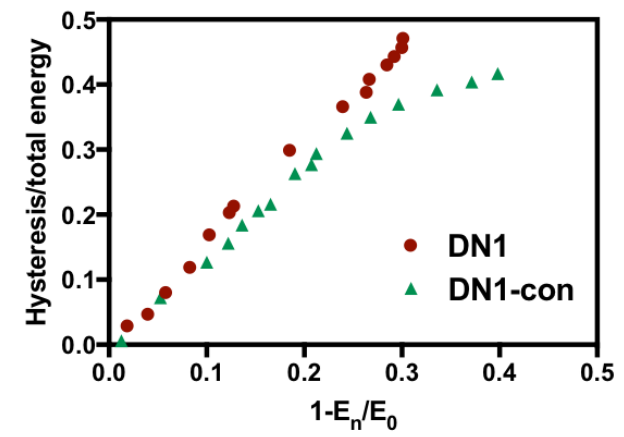

Fig. 4. Hysteresis in cyclic loading/unloading of DN1 (A), and DN1-con (B) in small strain range; (C) Fractional loss of Young's modulus of DN1 and DN1-con as a function of strain; (D) Fractional hysteresis as a functional of fractional loss in modulus loss for DN1 and DN1-con.

strands (36). RSE suppresses this loss in modulus; greater strains are required to trigger a measurable change in modulus in DN1 than DN1-con, and the loss in modulus in DN1 consistently lags than observed in DN1-con as a function of strain (Figure 4C). RSE should also alter the correlation between energy dissipation and change is modulus, by allowing dissipation without chain scission. As seen in Figure 4D, DN1 exhibits greater hysteresis per loss in modulus than DN1-con. The difference is greatest at the early stages of loading (lower strains), where the ratio of extended but unbroken strands to broken strands is expected to be largest.

Looking forward, the concept of reactive chain extension demonstrated here has numerous implications for network design. First, reactive chain extension occurs only at large strains and only at the specific molecular locations that are about to break without it. Unlike the incorporation of weak, reversible cross-linking or noncovalent interstrand interactions, the enhancement does not come with a concomitant increase in the viscous modulus of the network. 
In other words, the energy dissipation is triggered only when needed to resist fracture and need not be overcome in routine functions of the material when fracture is not imminent. Second, the property enhancements are achieved within networks that otherwise have identical structure and modulus to their non-mechanophore controls, meaning that reactive chain extension might be introduced into at least some materials whose properties are already optimized for a given function. We note that the magnitude of the effect seen here is large enough that, if it were translated to fatigue-prone materials, it would have a substantial impact on material life cycle. The correlation between fatigue life of rubbery materials and $T$ has been appreciated for some time $(35,37)$, with scaling dependences of $T^{2}$ noted (38). Even greater property enhancements should be obtainable in the future through the optimization of mechanophore design, polymerization strategy, and multi-network architecture. Notably, relative to control DN gels, RSE provides this fatigue resistance while also suppressing the loss in modulus that accompanies energy dissipation. Third, prior examples of mechanochemically induced changes in macroscopic physical properties involve either activation in solution $(21,39)$, irreversible plastic deformation $(40,41)$, and/or the presence of reagents beyond the initial network $(11,39,41)$; we are not aware of direct correlations of macroscopic properties and mechanochemical response within an intact strand. Mechanophore designs offer an increasing range of responses (42), and the chemical changes that accompany reactive chain extension might be designed to produce additional, desirable responses. For example, the ring opening of mechanophores similar to those employed here can be reversed photochemically (43), offering the potential for new types of molecular-scale repair that could further extend material lifetime. Finally, whereas reactive chain extension is demonstrated here in the context of DN hydrogels, single chain response should also contribute to the properties of single networks, elastomers, and polymer composites. The investigation of this rich structure-activity space, including the interplay of molecular response and network architecture, presents a rich set of opportunities and challenges that span synthetic, physical, theoretical, and analytical aspects of polymer chemistry. 
A Toughening DN by reactive strand extension

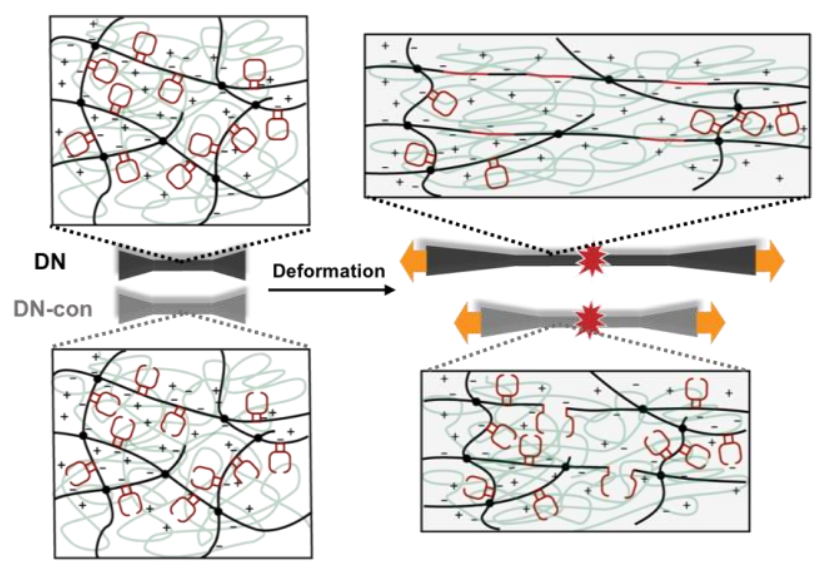

B Chemical designs of polyelectrolytes in first network

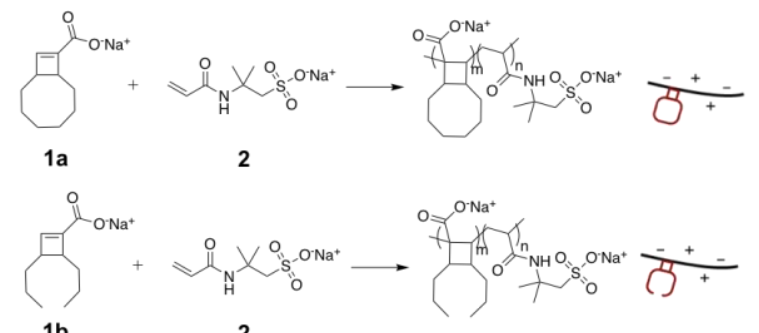

$1 \mathrm{~b}$

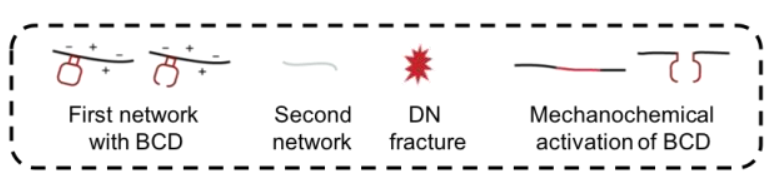

Fig. 1. The reactive strand extension (RSE) concept in double network hydrogels (strand scission events are not shown). (A) Schematic of double networks RSE and an analogous control polymer. (B) Chemical structures of the first network co-polymers employed in this work.

A Mechanochemical reactions

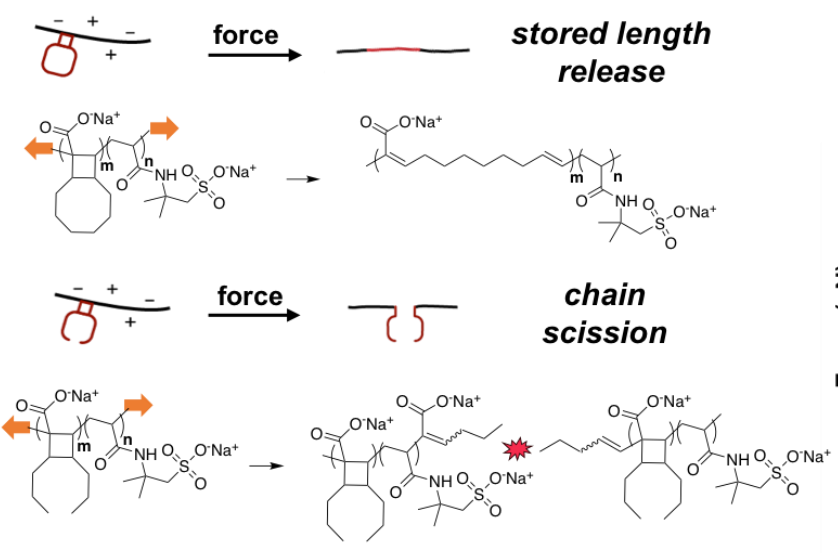

B
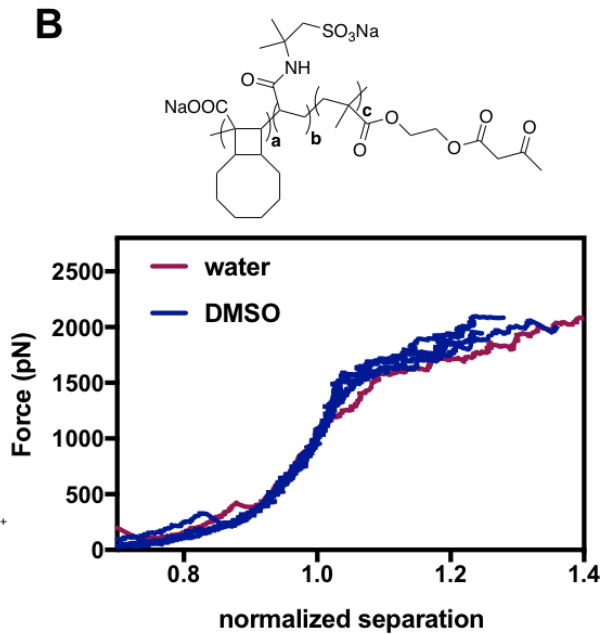

Fig. 2. Mechanochemical reactions driving RSE. (A) Mechanically coupled [2+2] cycloreversion leads to chain extension in [6.2.0]bicyclodecane mechanophores and to chain scission in analogous acyclic cyclobutane mechanophores. (B) Representative single molecule force-extension curves of linear RSE co-polymers in water and DMSO. 
Table 1. Young's modulus $(E)$, nominal strain, nominal stress, and input energy density at break ( $\varepsilon_{b}, \sigma_{b}$, and $W_{\mathrm{b}}$, respectively) of unnotched films in unconstrained uniaxial tension, and crack propagation strain $\left(\varepsilon_{\boldsymbol{p}}\right)$ and tearing energy $(T)$ of films in a single-edge notched geometry; Tearing energy of PAAm gel is listed as reported (44).

\begin{tabular}{ccccccc} 
& $\boldsymbol{E}(\mathbf{k P a})$ & $\boldsymbol{\varepsilon}_{\boldsymbol{b}}$ & $\boldsymbol{\sigma}_{\boldsymbol{b}}(\mathbf{M P a})$ & $\boldsymbol{W}_{\mathbf{b}}\left(\mathbf{M J} \mathbf{~ m}^{-\mathbf{3}}\right)$ & $\boldsymbol{\varepsilon}_{\boldsymbol{p}}$ & $\boldsymbol{T}\left(\mathbf{J ~ m}^{-\mathbf{2}}\right)$ \\
\hline $\mathbf{D N 1}$ & $288 \pm 4$ & 10.0 & 0.92 & 6.4 & 2.3 & $2840 \pm 103$ \\
$\mathbf{D N 1 - c o n}$ & $290 \pm 8$ & 4.9 & 0.61 & 2.3 & 1.7 & $1419 \pm 52$ \\
$\mathbf{D N 2}$ & $243 \pm 5$ & 7.0 & 0.64 & 2.6 & 1.3 & $554 \pm 40$ \\
DN2-con & $249 \pm 13$ & 4.7 & 0.54 & 1.6 & 0.8 & $276 \pm 10$ \\
PAAm & $57 \pm 6$ & 5.0 & 0.18 & 0.4 & $/$ & $\sim 10$
\end{tabular}


A

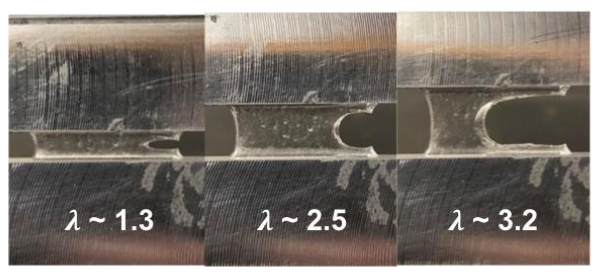

C

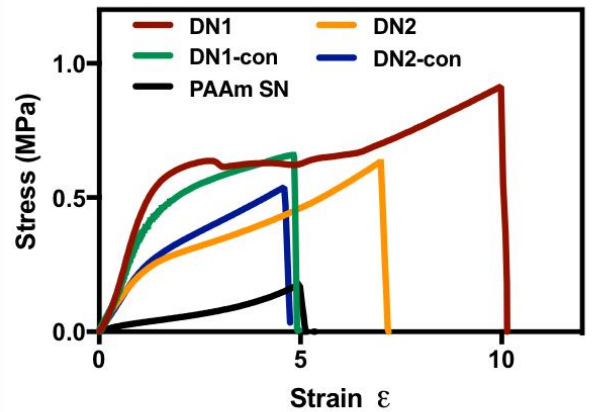

D

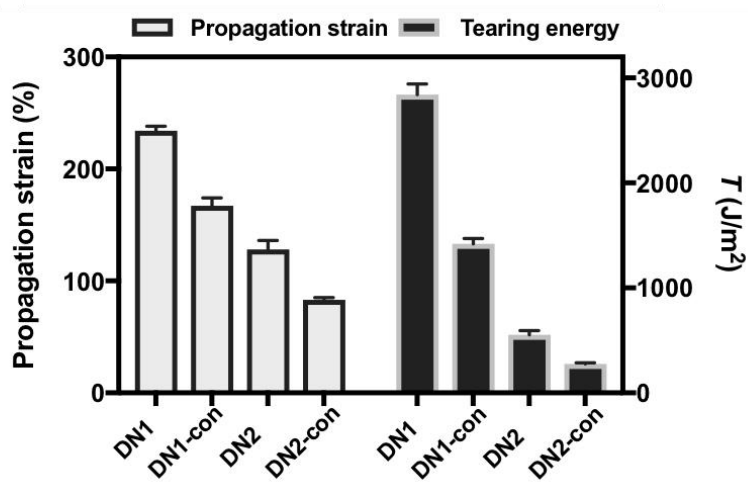

B

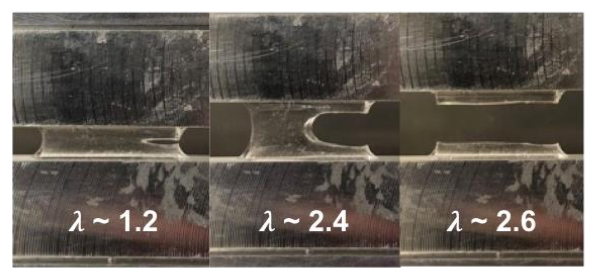

E
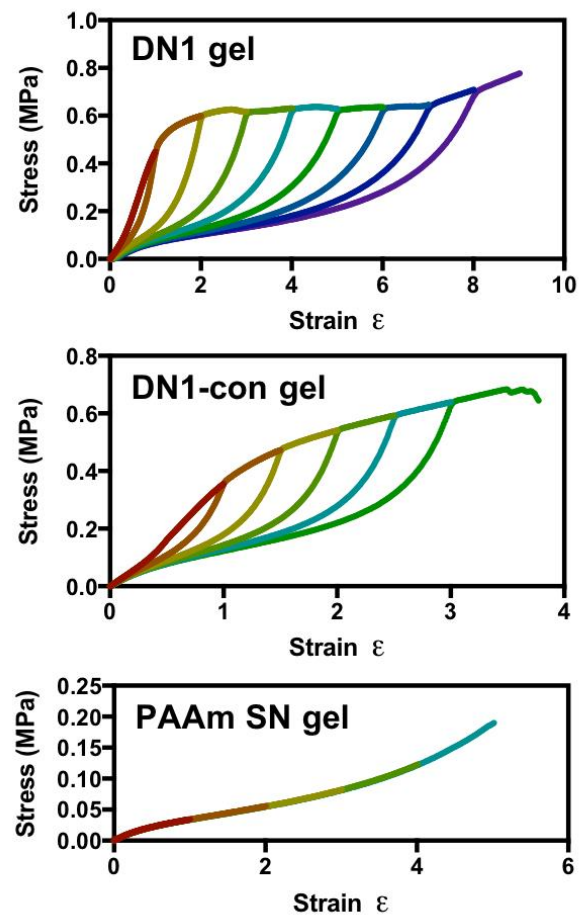

Fig. 3. Response of DNs to uniaxial tension. (A) Single-edge notched DN1 at stretch ratios $(\mathrm{mm} / \mathrm{mm})$ of 1.3, 2.5, and 3.2. (B) Single-edge notched DN1-con at stretch ratios $(\mathrm{mm} / \mathrm{mm})$ of 1.2, 2.4, and 2.6. (C) Representative stress-strain curves of unnotched DN films and a single PAAm network prepared identically to the second network of the DNs. (D) Propagation strain and tear energy of DNs. (E) Hysteresis in cyclic loading/unloading of DN1, DN1-con, and a single PAAm network prepared identically to the second network of the DNs. 
A

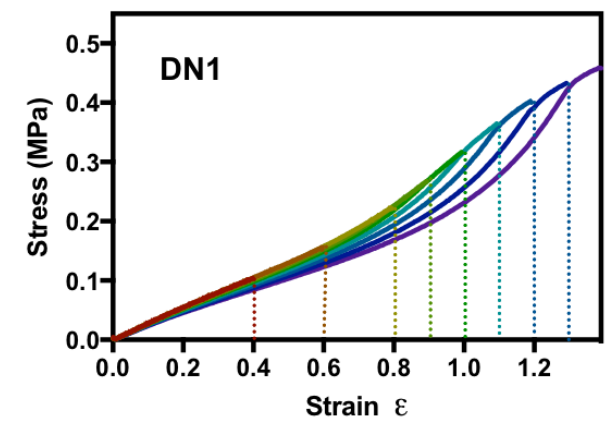

C

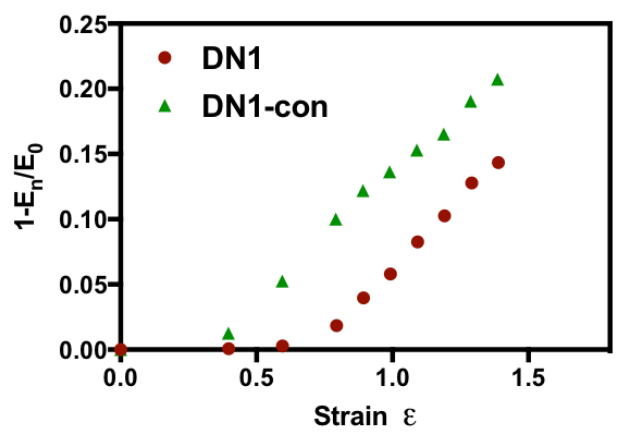

B

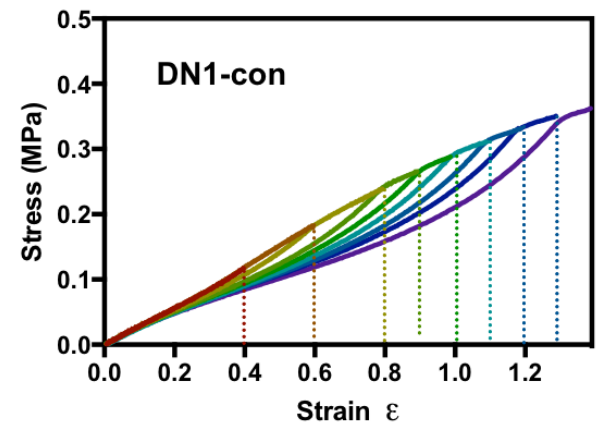

D

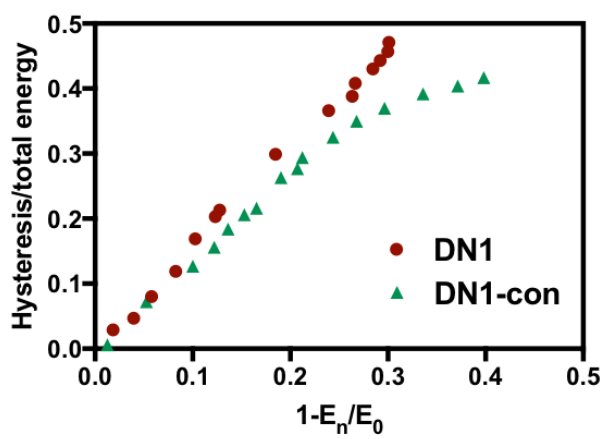

Fig. 4. Hysteresis in cyclic loading/unloading of DN1 (A), and DN1-con (B) in small strain range; (C) Fractional loss of Young's modulus of DN1 and DN1-con as a function of strain; (D) Fractional hysteresis as a functional of fractional loss in modulus loss for DN1 and DN1-con. 


\section{References and Notes:}

1. M. M. Caruso et al., Mechanically-Induced Chemical Changes in Polymeric Materials. Chemical Reviews 109, 5755-5798 (2009).

2. S. Lin et al., Anti-fatigue-fracture hydrogels. Science Advances 5, eaau8528 (2019).

3. U.S. Tire Manufacturers Association, (USTMA), "2015 U.S. Scrap Tire Management Summary” (USTMA, 2017; www.ustires.org/sites/default/files/MAR_028_USTMA.pdf)

4. I. McDermott, Meniscal tears, repairs and replacement: their relevance to osteoarthritis of the knee. British Journal of Sports Medicine 45, 292 (2011).

5. T. De Coninck et al., Two-Year Follow-up Study on Clinical and Radiological Outcomes of Polyurethane Meniscal Scaffolds. The American Journal of Sports Medicine 41, 64-72 (2012).

6. S. E. Root, S. Savagatrup, A. D. Printz, D. Rodriquez, D. J. Lipomi, Mechanical Properties of Organic Semiconductors for Stretchable, Highly Flexible, and Mechanically Robust Electronics. Chemical Reviews 117, 6467-6499 (2017).

7. X. Zhao, Multi-scale multi-mechanism design of tough hydrogels: building dissipation into stretchy networks. Soft Matter 10, 672-687 (2014).

8. X. Zhao, Designing toughness and strength for soft materials. Proceedings of the National Academy of Sciences 114, 8138 (2017).

9. E. Ducrot, Y. Chen, M. Bulters, R. P. Sijbesma, C. Creton, Toughening Elastomers with Sacrificial Bonds and Watching Them Break. Science 344, 186-189 (2014).

10. J.-Y. Sun et al., Highly stretchable and tough hydrogels. Nature 489, 133 (2012).

11. T. Matsuda, R. Kawakami, R. Namba, T. Nakajima, J. P. Gong, Mechanoresponsive selfgrowing hydrogels inspired by muscle training. Science 363, 504 (2019).

12. H. J. Zhang et al., Tough Physical Double-Network Hydrogels Based on Amphiphilic Triblock Copolymers. Advanced Materials 28, 4884-4890 (2016).

13. J. Hentschel, A. M. Kushner, J. Ziller, Z. Guan, Self-Healing Supramolecular Block Copolymers. Angewandte Chemie International Edition 51, 10561-10565 (2012).

14. J. Fang et al., Forced protein unfolding leads to highly elastic and tough protein hydrogels. Nature Communications 4, 2974 (2013).

15. J. Chung, A. M. Kushner, A. C. Weisman, Z. Guan, Direct correlation of single-molecule properties with bulk mechanical performance for the biomimetic design of polymers. Nature Materials 13, 1055-1062 (2014).

16. H. Lei et al., Stretchable hydrogels with low hysteresis and anti-fatigue fracture based on polyprotein cross-linkers. Nature Communications 11, 4032 (2020).

17. Z. Wang et al., Stretchable materials of high toughness and low hysteresis. Proceedings of the National Academy of Sciences 116, 5967 (2019).

18. S. Garcia-Manyes, A. E. M. Beedle, Steering chemical reactions with force. Nature Reviews Chemistry 1, 0083 (2017).

19. S. L. Craig, Up another rung. Nature Chemistry 9, 1154-1155 (2017).

20. Y. Tian et al., A Polymer with Mechanochemically Active Hidden Length. Journal of the American Chemical Society 142, 18687-18697 (2020).

21. Z. Chen et al., Mechanochemical unzipping of insulating polyladderene to semiconducting polyacetylene. Science 357, 475 (2017). 
22. S. Wang, S. Panyukov, M. Rubinstein, S. L. Craig, Quantitative Adjustment to the Molecular Energy Parameter in the Lake-Thomas Theory of Polymer Fracture Energy. Macromolecules 52, 2772-2777 (2019).

23. G. J. Lake, A. G. Thomas, The Strength of Highly Elastic Materials. Proc. R. Soc. Lond. A, 300, 108-119 (1967).

24. B. H. Bowser, C.-H. Ho, S. L. Craig, High Mechanophore Content, Stress-Relieving Copolymers Synthesized via RAFT Polymerization. Macromolecules 52, 9032-9038 (2019).

25. W. Denissen et al., Chemical control of the viscoelastic properties of vinylogous urethane vitrimers. Nature Communications 8, 14857 (2017).

26. J. J. Lessard et al., Block Copolymer Vitrimers. Journal of the American Chemical Society 142, 283-289 (2020).

27. J. Wang, T. B. Kouznetsova, R. Boulatov, S. L. Craig, Mechanical gating of a mechanochemical reaction cascade. Nature Communications 7, 13433 (2016).

28. M. F. Pill et al., Mechanochemical Cycloreversion of Cyclobutane Observed at the Single Molecule Level. Chemistry - A European Journal 22, 12034-12039 (2016).

29. I. M. Klein, C. C. Husic, D. P. Kovács, N. J. Choquette, M. J. Robb, Validation of the CoGEF Method as a Predictive Tool for Polymer Mechanochemistry. Journal of the American Chemical Society 142, 16364-16381 (2020).

30. J. P. Gong, Y. Katsuyama, T. Kurokawa, Y. Osada, Double-Network Hydrogels with Extremely High Mechanical Strength. Advanced Materials 15, 1155-1158 (2003).

31. J. P. Gong, Why are double network hydrogels so tough? Soft Matter 6, 2583-2590 (2010).

32. H. R. Brown, A Model of the Fracture of Double Network Gels. Macromolecules 40, 3815-3818 (2007).

33. T. Nakajima, T. Kurokawa, S. Ahmed, W.-1. Wu, J. P. Gong, Characterization of internal fracture process of double network hydrogels under uniaxial elongation. Soft Matter $\mathbf{9}$, 1955-1966 (2013).

34. M. Bacca, C. Creton, R. M. McMeeking, A Model for the Mullins Effect in Multinetwork Elastomers. Journal of Applied Mechanics 84, (2017).

35. G. J. Lake, P. B. Lindley, Cut growth and fatigue of rubbers. II. Experiments on a noncrystallizing rubber. Journal of Applied Polymer Science 8, 707-721 (1964).

36. T.-T. Mai, T. Matsuda, T. Nakajima, J. P. Gong, K. Urayama, Distinctive Characteristics of Internal Fracture in Tough Double Network Hydrogels Revealed by Various Modes of Stretching. Macromolecules 51, 5245-5257 (2018).

37. G. J. Lake, P. B. Lindley, The mechanical fatigue limit for rubber. Journal of Applied Polymer Science 9, 1233-1251 (1965).

38. A. G. Thomas, Rupture of rubber. V. Cut growth in natural rubber vulcanizates. Journal of Polymer Science 31, 467-480 (1958).

39. Z. Wang, J. Ayarza, A. P. Esser-Kahn, Mechanically Initiated Bulk-Scale Free-Radical Polymerization. Angewandte Chemie International Edition 58, 12023-12026 (2019).

40. A. L. Black, J. A. Orlicki, S. L. Craig, Mechanochemically triggered bond formation in solid-state polymers. Journal of Materials Chemistry 21, 8460-8465 (2011).

41. R. T. M. Jakobs, S. Ma, R. P. Sijbesma, Mechanocatalytic Polymerization and CrossLinking in a Polymeric Matrix. ACS Macro Letters 2, 613-616 (2013). 
42. M. A. Ghanem et al., The role of polymer mechanochemistry in responsive materials and additive manufacturing. Nature Reviews Materials, (2020).

43. Z. S. Kean, A. L. Black Ramirez, Y. Yan, S. L. Craig, Bicyclo[3.2.0]heptane Mechanophores for the Non-scissile and Photochemically Reversible Generation of Reactive Bis-enones. Journal of the American Chemical Society 134, 12939-12942 (2012).

44. Y. Tanaka et al., Determination of Fracture Energy of High Strength Double Network Hydrogels. The Journal of Physical Chemistry B 109, 11559-11562 (2005).

45. J. Wang et al., Inducing and quantifying forbidden reactivity with single-molecule polymer mechanochemistry. Nature Chemistry 7, 323 (2015).

46. E. L. Florin et al., Sensing specific molecular interactions with the atomic force microscope. Biosensors and Bioelectronics 10, 895-901 (1995).

47. S. Ahmed, T. Nakajima, T. Kurokawa, M. Anamul Haque, J. P. Gong, Brittle-ductile transition of double network hydrogels: Mechanical balance of two networks as the key factor. Polymer 55, 914-923 (2014).

48. M.-A. B. Kruft, L. H. Koole, A Convenient Method To Measure Monomer Reactivity Ratios. Application to Synthesis of Polymeric Biomaterials Featuring Intrinsic Radiopacity. Macromolecules 29, 5513-5519 (1996).

49. J. Undin, T. Illanes, A. Finne-Wistrand, A.-C. Albertsson, Random introduction of degradable linkages into functional vinyl polymers by radical ring-opening polymerization, tailored for soft tissue engineering. Polymer Chemistry 3, 1260-1266 (2012).

50. D. W. Behnken, Estimation of copolymer reactivity ratios: An example of nonlinear estimation. Journal of Polymer Science Part A: General Papers 2, 645-668 (1964).

51. M. Dube, R. A. Sanayei, A. Penlidis, K. F. O'Driscoll, P. M. Reilly, A microcomputer program for estimation of copolymerization reactivity ratios. Journal of Polymer Science Part A: Polymer Chemistry 29, 703-708 (1991).

52. C. O'Connor, Acidic and basic amide hydrolysis. Quarterly Reviews, Chemical Society 24, 553-564 (1970).

53. T. B. Kouznetsova, J. Wang, S. L. Craig, Combined Constant-Force and ConstantVelocity Single-Molecule Force Spectroscopy of the Conrotatory Ring Opening Reaction of Benzocyclobutene. ChemPhysChem 18, 1486-1489 (2017).

54. S. B. Smith, Y. Cui, C. Bustamante, Overstretching B-DNA: The Elastic Response of Individual Double-Stranded and Single-Stranded DNA Molecules. Science 271, 795 (1996).

55. X. Hu, M. E. McFadden, R. W. Barber, M. J. Robb, Mechanochemical Regulation of a Photochemical Reaction. Journal of the American Chemical Society 140, 14073-14077 (2018).

56. X. Hu, T. Zeng, C. C. Husic, M. J. Robb, Mechanically Triggered Small Molecule Release from a Masked Furfuryl Carbonate. Journal of the American Chemical Society 141, 15018-15023 (2019).

57. Y. Lin, M. H. Barbee, C.-C. Chang, S. L. Craig, Regiochemical Effects on Mechanophore Activation in Bulk Materials. Journal of the American Chemical Society 140, 15969-15975 (2018). 
58. G. R. Gossweiler, T. B. Kouznetsova, S. L. Craig, Force-Rate Characterization of Two Spiropyran-Based Molecular Force Probes. Journal of the American Chemical Society 137, 6148-6151 (2015).

59. Y. Lin, T. B. Kouznetsova, S. L. Craig, Mechanically Gated Degradable Polymers. Journal of the American Chemical Society 142, 2105-2109 (2020).

60. T. Matsuda, T. Nakajima, J. P. Gong, Fabrication of Tough and Stretchable Hybrid Double-Network Elastomers Using Ionic Dissociation of Polyelectrolyte in Nonaqueous Media. Chemistry of Materials 31, 3766-3776 (2019).

61. A. Arora et al., Fracture of Polymer Networks Containing Topological Defects. Macromolecules 53, 7346-7355 (2020). 